\title{
MATHEMATICAL MODEL OF THE SHIP MANOEUVRING
}

\author{
Dan Obreja \\ "Dunarea de Jos" University of Galati, \\ Faculty of Naval Architecture, Galati, \\ Domneasca Street, No. 47, 800008, Romania, \\ E-mail:dan.obreja@ugal.ro
}

\begin{abstract}
Starting with the initial design phase, the knowledge of the ship manoeuvring performance is an important problem. One of the methods to evaluate the manoeuvring characteristics is based on the time domain simulation of the standard manoeuvring tests. A typical mathematical model that may be used to determine the manoeuvring characteristics of the ships is presented in this paper.
\end{abstract}

Keywords: ship motion, manoeuvring, mathematical model.

\section{INTRODUCTION}

The knowledge of the ship manoeuvring performance is an important problem, starting with the initial design phase ([3], [5]).

The ship manoeuvring concept comprises [2]: course keeping, manoeuvring and speed changing (including ship stopping).

Ussualy, the ship manoeuvring performance can be evaluated on the basis of the time domain simulation of the standard manoeuvring tests ([1], [3], [4]). In this case, a mathematical model of the ship motion in horizontal plane must be developed and the total forces and moments acting on the hull must be calculated.

Two distinct groups of the control devices can be defined in order to control the ship motion in waterline plane:

$>$ Active control devices use the own energy (propelllers, thrusters, steering nozzles);

$>$ Passive control devices absorbe the water energy from the propeller race (rudders).

The control devices produce the control forces and moments acting on the ship.
At the same time, the hydrodynamics (reactions) forces and moments due to the ship motions are generated. The total external forces and moments include both the control and the hydrodynamics forces and moments.

In order to study the ship motion of a rigid body with six degree of freedom, the deep, unrestricted and calm water assumptions are considered.

Fig.1 [4] shows the fixed reference system $\mathrm{O}_{0} \mathrm{x}_{0} \mathrm{y}_{0} \mathrm{z}_{0}$ and also the moving reference system Oxyz, attached to the ship.

The origin $\mathrm{O}$ of the fixed coordinate system is considered at the midship section, on the symmetry plane $(\mathrm{Oxz})$.

The velocity vector $\bar{v}_{0}(\mathrm{u}, \mathrm{v}, \mathrm{w})$ is tangent to the ship trajectory, the angular speed is noted with $\bar{\omega}(\mathrm{p}, \mathrm{q}, \mathrm{r})$ and the coordinates of the center of gravity are $\mathrm{G}\left(\mathrm{x}_{\mathrm{G}}, \mathrm{y}_{\mathrm{G}}, \mathrm{z}_{\mathrm{G}}\right)$.

The heeding angle $\psi$ refers to the direction of the ship's longitudinal axis $\mathrm{Ox}$ with respect to the fixed axix $\mathrm{O}_{0} \mathrm{x}_{0}$. Also, the drift angle $\beta$ refers to the direction of the ship's longitudinal axis Ox with respect to the direction of the velocity vector $\bar{v}_{0}$. 
The components of the total external forces and moments are noted with $\bar{F}$ (X, Y, $\mathrm{Z})$ and $\bar{M}_{t}(\mathrm{~K}, \mathrm{M}, \mathrm{N})$ respectively.

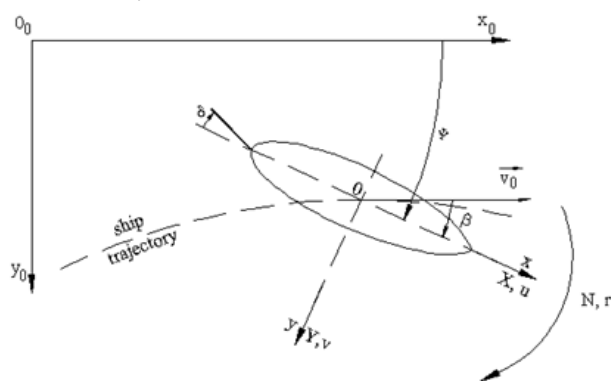

Fig. 1. Reference systems

The general problem of the ship motion equations with six degree of freedom is developed in the next chapter.

\section{SHIP MOTION EQUATIONS}

The ship motion of a body with six degree of freedom may be determined on the basis of linear and angular momentum theorems [6].

For $\mathrm{N}$ number of small particles with mass $\mathrm{m}_{\mathrm{i}}$ and speed $\bar{v}_{i}$, the linear momentum theorem may be written under the following form

$$
\sum_{i=1}^{N} \bar{F}_{i}=\sum_{i=1}^{N} \frac{d}{d t}\left(m_{i} \cdot \bar{v}_{i}\right)
$$

where $\bar{F}_{i}$ is the total external force acting on the particle $i$. The speed $\bar{v}_{i}$ has the expression

$$
\bar{v}_{i}=\bar{v}_{0}+\bar{\omega} \times \bar{r}_{i}
$$

where $\bar{r}_{i}\left(\mathrm{x}_{\mathrm{i}}, \mathrm{y}_{\mathrm{i}}, \mathrm{Z}_{\mathrm{i}}\right)$ is the vector radius of the particle $\mathrm{i}$.

Using the relation (2), the equation (1) becomes

$$
\sum_{i=1}^{N} \bar{F}_{i}=m \cdot \frac{\partial \bar{v}_{0}}{\partial t}+\frac{d}{d t}\left(\bar{\omega} \times \sum_{i=1}^{N} m_{i} \cdot \bar{r}_{i}\right)
$$

where $m=\sum_{i=1}^{N} m_{i}$ is the ship mass.
If $\bar{r}_{G}\left(\mathrm{x}_{\mathrm{G}}, \mathrm{y}_{\mathrm{G}}, \mathrm{Z}_{\mathrm{G}}\right)$ is the vector radius of the centre of gravity and the next relation is considered

$$
m \cdot \bar{r}_{G}=\sum_{i=1}^{N}\left(m_{i} \cdot \bar{r}_{i}\right)
$$

then, the following successive transformations may be written

$$
\begin{aligned}
& \bar{F}=\sum_{i=1}^{N} \bar{F}_{i}=m \cdot \frac{\partial \bar{v}_{0}}{\partial t}+m \frac{d}{d t}\left(\bar{\omega} \times \bar{r}_{G}\right)= \\
& =m\left(\frac{\partial \bar{v}_{0}}{\partial t}+\frac{d \bar{\omega}}{d t} \times \bar{r}_{G}+\bar{\omega} \times \frac{d \bar{r}_{G}}{d t}\right)= \\
& =m\left[\frac{\partial \bar{v}_{0}}{\partial t}+\frac{d \bar{\omega}}{d t} \times \bar{r}_{G}+\bar{\omega} \times\left(\bar{v}_{0}+\bar{\omega} \times \bar{r}_{G}\right)\right]= \\
& =m\left[\frac{\partial \bar{v}_{0}}{\partial t}+\frac{d \bar{\omega}}{d t} \times \bar{r}_{G}+\bar{\omega} \times \bar{v}_{0}+\bar{\omega} \times\left(\bar{\omega} \times \bar{r}_{G}\right)\right]
\end{aligned}
$$

On the basis of the next forms of the vector products and triple vector product

$$
\begin{aligned}
& \frac{d \bar{\omega}}{d t} \times \bar{r}_{G}=\left|\begin{array}{ccc}
\bar{i} & \bar{j} & \bar{k} \\
\frac{d p}{d t} & \frac{d q}{d t} & \frac{d r}{d t} \\
x_{G} & y_{G} & z_{G}
\end{array}\right|= \\
& =\left(\frac{d q}{d t} \cdot z_{G}-\frac{d r}{d t} \cdot y_{G}\right) \bar{i}+ \\
& +\left(\frac{d r}{d t} \cdot x_{G}-\frac{d p}{d t} \cdot z_{G}\right) \bar{j}+ \\
& +\left(\frac{d p}{d t} \cdot y_{G}-\frac{d q}{d t} \cdot x_{G}\right) \bar{k}
\end{aligned}
$$

$\bar{\omega} \times \bar{v}_{0}=\left|\begin{array}{ccc}\bar{i} & \bar{j} & \bar{k} \\ p & q & r \\ u & v & w\end{array}\right|=$

$$
=(q \cdot w-r \cdot v) \bar{i}+(r \cdot u-p \cdot w) \bar{j}+
$$$$
+(p \cdot v-q u) \bar{k}
$$

$$
\bar{\omega} \times \bar{r}_{G}=\left|\begin{array}{lll}
\bar{i} & \bar{j} & \bar{k} \\
p & q & r \\
x_{G} & y_{G} & z_{G}
\end{array}\right|=
$$


$=\left(q \cdot z_{G}-r \cdot y_{G}\right) \bar{i}+\left(r \cdot x_{G}-p \cdot z_{G}\right) \bar{j}+$

$+\left(p \cdot y_{G}-q \cdot x_{G}\right) \bar{k}$

$\bar{\omega} \times\left(\bar{\omega} \times \bar{r}_{G}\right)=$

$=\left[p\left(q \cdot y_{G}+r \cdot z_{G}\right)-\left(q^{2}+r^{2}\right) x_{G}\right] \bar{i}+$

$+\left[q\left(r \cdot z_{G}+p \cdot x_{G}\right)-\left(r^{2}+p^{2}\right) \cdot y_{G}\right] \bar{j}+$

$+\left[r\left(p \cdot x_{G}+q \cdot y_{G}\right)-\left(p^{2}+q^{2}\right) \cdot z_{G}\right] \bar{k}$

the resulting three linear momentum equations may be obtained

$$
\begin{aligned}
& X=m \cdot\left[\frac{\partial u}{\partial t}+q w-r v+\frac{d q}{d t} z_{G}-\frac{d r}{d t} y_{G}+\right. \\
& \left.+\left(q y_{G}+r z_{G}\right) p-\left(q^{2}+r^{2}\right) x_{G}\right] \\
& Y=m \cdot\left[\frac{\partial v}{\partial t}+r u-p w+\frac{d r}{d t} x_{G}-\frac{d p}{d t} z_{G}+\right. \\
& \left.+\left(r z_{G}+p x_{G}\right) q-\left(r^{2}+p^{2}\right) y_{G}\right] \\
& Z=m \cdot\left[\frac{\partial w}{\partial t}+p v-q u+\frac{d p}{d t} y_{G}-\frac{d q}{d t} x_{G}+\right. \\
& \left.+\left(p x_{G}+q y_{G}\right) r-\left(p^{2}+q^{2}\right) z_{G}\right]
\end{aligned}
$$

The angular momentum theorem may be written under the form

$$
\sum_{i=1}^{N}\left(\bar{M}_{i}+\bar{r}_{i} \times \bar{F}_{i}\right)=\sum_{i=1}^{N} \bar{r}_{i} \times \frac{d}{d t}\left(m_{i} \cdot \bar{v}_{i}\right)
$$

where $\bar{M}_{i}$ is the total external moment acting on the particle i.

On the basis of successive transformations, the following expressions may be obtained

$$
\begin{aligned}
& \sum_{i=1}^{N}\left(\bar{M}_{i}+\bar{r}_{i} \times \bar{F}_{i}\right)=\sum_{i=1}^{N} m_{i} \bar{r}_{i} \times \frac{d}{d t}\left(\bar{v}_{0}+\bar{\omega} \times \bar{r}_{i}\right)= \\
& =\sum_{i=1}^{N} m_{i} \bar{r}_{i} \times\left(\frac{\partial \bar{v}_{0}}{\partial t}+\frac{d \bar{\omega}}{d t} \times \bar{r}_{i}+\bar{\omega} \times \frac{d \bar{r}_{i}}{d t}\right)= \\
& =m \cdot \bar{r}_{G} \times \frac{\partial \bar{v}_{0}}{\partial t}+\sum_{i=1}^{N} m_{i} \bar{r}_{i} \times\left(\frac{\partial \bar{\omega}}{\partial t} \times \bar{r}_{i}\right)+ \\
& +\sum_{i=1}^{N} m_{i} \bar{r}_{i} \times\left[\bar{\omega} \times\left(\bar{v}_{0}+\bar{\omega} \times \bar{r}_{i}\right)\right]= \\
& =m \cdot \bar{r}_{G} \times \frac{\partial \bar{v}_{0}}{\partial t}+\sum_{i=1}^{N} m_{i} \bar{r}_{i} \times\left(\frac{\partial \bar{\omega}}{\partial t} \times \bar{r}_{i}\right)+ \\
& +m \cdot \bar{r}_{G} \times\left(\bar{\omega} \times \bar{v}_{0}\right)+\sum_{i=1}^{N} m_{i} \bar{r}_{i} \times\left[\bar{\omega} \times\left(\bar{\omega} \times \bar{r}_{i}\right)\right]=
\end{aligned}
$$

$$
\begin{aligned}
& =m \bar{r}_{G} \times\left(\frac{\partial \bar{v}_{0}}{\partial t}+\bar{\omega} \times \bar{v}_{0}\right)+\sum_{i=1}^{N} m_{i} \bar{r}_{i} \times\left(\frac{\partial \bar{\omega}}{\partial t} \times \bar{r}_{i}\right)+ \\
& +\sum_{i=1}^{N} m_{i} \bar{r}_{i} \times\left[\bar{\omega} \times\left(\bar{\omega} \times \bar{r}_{i}\right)\right]
\end{aligned}
$$

The following vector products and triple vector products may be developed

$\frac{\partial \bar{\omega}}{\partial t} \times \bar{r}_{i}=\left|\begin{array}{ccc}\bar{i} & \bar{j} & \bar{k} \\ \frac{\partial p}{\partial t} & \frac{\partial q}{\partial t} & \frac{\partial r}{\partial t} \\ x_{i} & y_{i} & z_{i}\end{array}\right|=$

$=\left(\frac{\partial q}{\partial t} z_{i}-\frac{\partial r}{\partial t} y_{i}\right) \bar{i}+\left(\frac{\partial r}{\partial t} x_{i}-\frac{\partial p}{\partial t} z_{i}\right) \bar{j}+$

$+\left(\frac{\partial p}{\partial t} y_{i}-\frac{\partial q}{\partial t} x_{i}\right) \bar{k}$

$\bar{r}_{i} \times\left(\frac{\partial \bar{\omega}}{\partial t} \times \bar{r}_{i}\right)=$

$=\left[\frac{\partial p}{\partial t}\left(y_{i}^{2}+z_{i}^{2}\right)-\left(y_{i} \frac{\partial q}{\partial t}+z_{i} \frac{\partial r}{\partial t}\right) x_{i}\right] \bar{i}+$

$+\left[\frac{\partial q}{\partial t}\left(x_{i}^{2}+z_{i}^{2}\right)-\left(x_{i} \frac{\partial p}{\partial t}+z_{i} \frac{\partial r}{\partial t}\right) y_{i}\right] \bar{j}+$

$+\left[\frac{\partial r}{\partial t}\left(x_{i}^{2}+y_{i}^{2}\right)-\left(x_{i} \frac{\partial p}{\partial t}+y_{i} \frac{\partial q}{\partial t}\right) z_{i}\right] \bar{k}$

$$
\begin{gathered}
\frac{\partial \bar{v}_{0}}{\partial t}+\bar{\omega} \times \bar{v}_{0}=\left(\frac{\partial u}{\partial t}+q w-r v\right) \bar{i}+ \\
+\left(\frac{\partial v}{\partial t}+r u-p w\right) \bar{j}+ \\
+\left(\frac{\partial w}{\partial t}+p v-q u\right) \bar{k} \\
\bar{\omega} \times\left(\bar{\omega} \times \bar{r}_{i}\right)=\left[p\left(q y_{i}+r z_{i}\right)-\left(q^{2}+r^{2}\right) x_{i} \bar{j}+\right. \\
+\left[q\left(r z_{i}+p x_{i}\right)-\left(r^{2}+p^{2}\right) y_{i}\right] \bar{j}+ \\
+\left[r\left(p x_{i}+q y_{i}\right)-\left(p^{2}+q^{2}\right) z_{i}\right] \bar{k}
\end{gathered}
$$

$\bar{r}_{i} \times\left[\bar{\omega} \times\left(\bar{\omega} \times \bar{r}_{i}\right)\right]=$

$=\left[\left(r^{2}-q^{2}\right) y_{i} z_{i}-p q x_{i} z_{i}+p r x_{i} y_{i}+r d\left(y_{i}^{2}-z_{i}^{2}\right)\right] \bar{i}+$

$+\left[\left(p^{2}-r^{2}\right) x_{i} z_{i}-r q x_{i} y_{i}+p q y_{i} z_{i}+r p\left(z_{i}^{2}-x_{i}^{2}\right)\right] j+$

$+\left[\left(q^{2}-p^{2}\right) x_{i} y_{i}-p r y_{i} z_{i}+q r x_{i} z_{i}+q p\left(x_{i}^{2}-y_{i}^{2}\right)\right] \bar{k}$ 


$$
\begin{aligned}
& m \bar{r}_{G} \times\left(\frac{\partial \bar{v}_{0}}{\partial t}+\bar{\omega} \times \bar{v}_{0}\right)= \\
& =m\left[y_{G}\left(\frac{\partial w}{\partial t}+p v-q u\right)-z_{G}\left(\frac{\partial v}{\partial t}+r u-p w\right)\right] \bar{i}+ \\
& +m\left[z_{G}\left(\frac{\partial u}{\partial t}+q w-r v\right)-x_{G}\left(\frac{\partial w}{\partial t}+p v-q u\right)\right] \bar{j}+ \\
& +m\left[x_{G}\left(\frac{\partial v}{\partial t}+r u-p w\right)-y_{G}\left(\frac{\partial u}{\partial t}+q w-r v\right)\right] \bar{k}
\end{aligned}
$$

On the basis of the matrix components of the ship mechanical inertia moments, defined by using the following relations

$$
\begin{gathered}
I=\left|\begin{array}{ccc}
I_{x x} & I_{x y} & I_{x z} \\
I_{y x} & I_{y y} & I_{y z} \\
I_{z x} & I_{z y} & I_{z z}
\end{array}\right| \\
I_{x x}=\sum_{i=1}^{N} m_{i}\left(y_{i}^{2}+z_{i}^{2}\right) \\
I_{y y}=\sum_{i=1}^{N} m_{i}\left(x_{i}^{2}+z_{i}^{2}\right) \\
I_{z z}=\sum_{i=1}^{N} m_{i}\left(x_{i}^{2}+y_{i}^{2}\right) \\
I_{x y}=I_{y x}=-\sum_{i=1}^{N} m_{i} x_{i} y_{i} \\
I_{x z}=I_{z x}=-\sum_{i=1}^{N} m_{i} x_{i} z_{i} \\
I_{y z}=I_{z y}=-\sum_{i=1}^{N} m_{i} y_{i} z_{i} .
\end{gathered}
$$

the next equivalent forms may be obtained

$$
\begin{gathered}
\sum_{i=1}^{N} m_{i} \bar{r}_{i} \times\left(\frac{\partial \bar{\omega}}{\partial t} \times \bar{r}_{i}\right)= \\
=\sum_{i=1}^{N} m_{i} \cdot\left[\frac{\partial p}{\partial t}\left(y_{i}^{2}+z_{i}^{2}\right)-\left(y_{i} \frac{\partial q}{\partial t}+z_{i} \frac{\partial r}{\partial t}\right) x_{i}\right] \bar{i}+ \\
+\sum_{i=1}^{N} m_{i} \cdot\left[\frac{\partial q}{\partial t}\left(x_{i}^{2}+z_{i}^{2}\right)-\left(x_{i} \frac{\partial p}{\partial t}+z_{i} \frac{\partial r}{\partial t}\right) y_{i}\right] \bar{j}+ \\
+\sum_{i=1}^{N} m_{i} \cdot\left[\frac{\partial r}{\partial t}\left(x_{i}^{2}+y_{i}^{2}\right)-\left(x_{i} \frac{\partial p}{\partial t}+y_{i} \frac{\partial q}{\partial t}\right) z_{i}\right] \bar{k}= \\
=\left(\frac{\partial p}{\partial t} I_{x x}+\frac{\partial q}{\partial t} I_{x y}+\frac{\partial r}{\partial t} I_{x z}\right) \bar{i}+
\end{gathered}
$$

$$
\begin{gathered}
+\left(\frac{\partial p}{\partial t} I_{y x}+\frac{\partial q}{\partial t} I_{y y}+\frac{\partial r}{\partial t} I_{y z}\right) \bar{j}+ \\
+\left(\frac{\partial p}{\partial t} I_{z x}+\frac{\partial q}{\partial t} I_{z y}+\frac{\partial r}{\partial t} I_{z z}\right) \bar{k} \\
\sum_{i=1}^{N} m_{i} \bar{r}_{i} \times\left[\bar{\omega} \times\left(\bar{\omega} \times \bar{r}_{i}\right)\right]= \\
=\left[\left(q^{2}-r^{2}\right) I_{y z}+p q I_{x z}-p r I_{x y}+r q\left(I_{z z}-I_{y y}\right)\right] \bar{i}+ \\
+\left[\left(r^{2}-p^{2}\right) I_{x z}+r q I_{x y}-p q I_{y z}+r p\left(I_{x x}-I_{z z}\right)\right] \bar{j}+ \\
+\left[\left(p^{2}-q^{2}\right) I_{x y}+p r I_{y z}-q r I_{x z}+q p\left(I_{y y}-I_{x x}\right)\right] \bar{k}
\end{gathered}
$$

As a consequence, the resulting three angular momentum equations may be determined

$$
\begin{aligned}
K & =\frac{\partial p}{\partial t} I_{x x}+\frac{\partial q}{\partial t} I_{x y}+\frac{\partial r}{\partial t} I_{x z}+ \\
& +r q\left(I_{z z}-I_{y y}\right)+\left(q^{2}-r^{2}\right) I_{y z}+p q I_{x z}-p r I_{x y}+ \\
& +m\left[y_{G}\left(\frac{\partial w}{\partial t}+p v-q u\right)-z_{G}\left(\frac{\partial v}{\partial t}+r u-p w\right)\right] \\
M & =\frac{\partial p}{\partial t} I_{y x}+\frac{\partial q}{\partial t} I_{y y}+\frac{\partial r}{\partial t} I_{y z}+ \\
& +p r\left(I_{x x}-I_{z z}\right)+\left(r^{2}-p^{2}\right) I_{x z}+q r I_{x y}-q p I_{y z}+ \\
& +m\left[z_{G}\left(\frac{\partial u}{\partial t}+q w-r v\right)-x_{G}\left(\frac{\partial w}{\partial t}+p v-q u\right)\right] \\
N & =\frac{\partial p}{\partial t} I_{z x}+\frac{\partial q}{\partial t} I_{z y}+\frac{\partial r}{\partial t} I_{z z}+ \\
& +p q\left(I_{y y}-I_{x x}\right)+\left(p^{2}-q^{2}\right) I_{x y}+p r I_{y z}-q r I_{x z}+ \\
& +m\left[x_{G}\left(\frac{\partial v}{\partial t}+r u-p w\right)-y_{G}\left(\frac{\partial u}{\partial t}+q w-r v\right)\right]
\end{aligned}
$$

The relations (10) and (24) constitute the differential equations system of the ship motion with six degree of freedom ([3], [6]).

\section{SHIP MOTION IN HORIZONTAL PLANE}

In the particular case of the ship manoeuvring in horizontal plane, only the surge, sway, yaw and sometimes the roll motion can be considered.

As a consequence, neglecting the crossinertia terms and assuming the ship symmetry related to the centerline plane, the follow- 
ing form of the ship motion equations may be written [3]

$$
\begin{aligned}
& X=m\left(\frac{\partial u}{\partial t}-r v-r^{2} \cdot x_{G}+p r z_{G}\right) \\
& Y=m\left(\frac{\partial v}{\partial t}+r u+\frac{d r}{d t} x_{G}-\frac{d p}{d t} z_{G}\right) \\
& K=\frac{\partial p}{\partial t} I_{x x}-m z_{G}\left(\frac{\partial v}{\partial t}+r u\right) \\
& N=\frac{\partial r}{\partial t} I_{z z}+m x_{G}\left(\frac{\partial v}{\partial t}+r u\right)
\end{aligned}
$$

A simplified form [3] may be obtained by neglecting both the roll motion influence $(\mathrm{K}=\mathrm{p}=0)$ and the small terms of second order $\left(\mathrm{r}^{2}=\mathrm{rv}=0\right)$

$$
\begin{gathered}
X=m\left(\frac{\partial u}{\partial t}\right) \\
Y=m\left(\frac{\partial v}{\partial t}+r u+\frac{d r}{d t} x_{G}\right) \\
N=\frac{\partial r}{\partial t} I_{z z}+m x_{G}\left(\frac{\partial v}{\partial t}+r u\right)
\end{gathered}
$$

The most important problem is to determine the forces $(\mathrm{X}, \mathrm{Y})$ and the yaw moment $(\mathrm{N})$ acting on the rigid body during the manoeuvring tests.

The external forces and moments represent the sum of the control $\left(\mathrm{X}_{\mathrm{c}}, \mathrm{Y}_{\mathrm{c}}, \mathrm{N}_{\mathrm{c}}\right)$ and the hydrodynamics $\left(\mathrm{X}_{\mathrm{rh}}, \mathrm{Y}_{\mathrm{rh}}, \mathrm{N}_{\mathrm{rh}}\right)$ forces and moments

$$
\begin{aligned}
& X=X_{c}+X_{r h} \\
& Y=Y_{c}+Y_{r h} \\
& N=N_{c}+N_{r h}
\end{aligned}
$$

The control forces and moments depend by the rudder deflection angle $\delta$

$$
\begin{aligned}
& X_{c}=f(\delta) \\
& Y_{c}=f(\delta) \\
& N_{c}=f(\delta)
\end{aligned}
$$

The hydrodynamics forces and moments depend by the speed (u,v), angular speed (r), acceleration $(\dot{u}, \dot{v})$ and angular acceleration $(\dot{r})$ components

$$
\begin{aligned}
& X_{r h}=f(u, \dot{u}) \\
& Y_{r h}=f(v, r, \dot{v}, \dot{r}) \\
& N_{r h}=f(r, v, \dot{r}, \dot{v})
\end{aligned}
$$

By using the Taylor series expansion and determining the hydrodynamics derivatives, the total external forces and moments may be obtained.

In the case of a linear hydrodynamic model, the following form of the ship motion equations may be written [3]

$$
\begin{aligned}
& X_{c}+X_{u} u+X_{\dot{u}} \dot{u}=m \dot{u} \\
& Y_{c}+Y_{v} v+Y_{r} r+Y_{\dot{v}} \dot{v}+Y_{\dot{r}} \dot{r}= \\
& =m\left(\dot{v}+r U+\dot{r} x_{G}\right) \\
& N_{c}+N_{v} v+N_{r} r+N_{\dot{v}} \dot{v}+N_{\dot{r}} \dot{r}= \\
& =I_{z z} \dot{r}+m x_{G}(\dot{v}+r U)
\end{aligned}
$$

where $\mathrm{U}$ is the ship speed.

Complex forms of the ship motion equations may be obtained in the case of the nonlinear hydrodynamics models.

\section{CONCLUDING REMARKS}

Starting with the initial design phase, the estimation of the ship manoeuvring performance is an important issue.

The ship manoeuvring performance can be determined on the basis of the time domain simulation of the standard manoeuvring tests.

In this paper, a mathematical model of the ship motion in horizontal plane was developed. The evaluation of the external forces and moments acting on the hull is the most important problem, which may be realised by using Taylor series expansion and hydrodynamics derivatives computation.

Practical evaluations of the ship manoeuvring characteristics may be performed on the basis of the mathematical model developed in this paper.

\section{Acknowledgements}

The paper was supported by the Research Centre of the Naval Architecture Faculty from "Dunarea de Jos" University of Galati, which is greatly acknowledged. 


\section{REFERENCES}

[1]. Abkowitz, M.A., "Lectures on Ship Hydrodynamics-Steering and Manoeuvrability", Report No. Hy-5, Hydro-and Aerodynamics Laboratory, Lyngby, Denmark, 1964.

[2]. Lewis, E.V., "Principles of Naval Architecture", Vol. III- "Motions in Waves and Controllability”, SNAME, USA, 1989.

[3]. Obreja, C.D., Crudu, L., Pacuraru, S., "Ship Manoeuvering”, Galati University Press, Galati, 2008 (in Romanian).

[4]. Obreja, C.D., Nabergoj, R., Crudu, L., Pacuraru, S., "Identification of hydrodynamic coefficients for manoeu- vring simulation model of a fishing vessel", Ocean Engineering, Vol. 37, Iss. 8-9, pp. 678-687, 2010.

[5]. Obreja, C.D., "Software platform for manoeuvrability performance estimation in initial ship design", The Annals of "Dunarea de Jos" University of Galati, Fascicle XI-Shipbuilding, pp. 1520, 2015.

[6]. Triantafyllou, M.S., Hover, F.S., "Maneuvering and Control of Marine Vehicles”, Department of Ocean Engineering, Massachusetts Institute of Technology Cambridge, Massachusetts, USA, 2002.

Paper received on November $9^{\text {th }}, 2019$ 